\title{
Fractional Differential Equations in Terms of Comparison Results and Lyapunov Stability with Initial Time Difference
}

\author{
Coşkun Yakar \\ Department of Mathematics, Gebze Institute of Technology, Gebze-Kocaeli 141-41400, Turkey \\ Correspondence should be addressed to Coşkun Yakar, cyakar@gyte.edu.tr
}

Received 16 September 2009; Revised 29 November 2009; Accepted 7 January 2010

Academic Editor: Dumitru Băleanu

Copyright (C 2010 Coşkun Yakar. This is an open access article distributed under the Creative Commons Attribution License, which permits unrestricted use, distribution, and reproduction in any medium, provided the original work is properly cited.

The qualitative behavior of a perturbed fractional-order differential equation with Caputo's derivative that differs in initial position and initial time with respect to the unperturbed fractionalorder differential equation with Caputo's derivative has been investigated. We compare the classical notion of stability to the notion of initial time difference stability for fractional-order differential equations in Caputo's sense. We present a comparison result which again gives the null solution a central role in the comparison fractional-order differential equation when establishing initial time difference stability of the perturbed fractional-order differential equation with respect to the unperturbed fractional-order differential equation.

\section{Introduction}

We have investigated that the stability of perturbed solution with respect to unperturbed solution with initial time difference of the nonlinear differential equations of fractionalorder. The differential operators are taken in the Riemann-Liouville and Caputo's sense and the initial conditions are specified according to Caputo's suggestion [1], thus allowing for interpretation in a physically meaningful way [2].

Lyapunov's second method is a standard technique used in the study of the qualitative behavior of differential systems [3-6] along with a comparison result $[2,7]$ that allows the prediction of behavior of a differential system when the behavior of the null solution of a comparison system is known. However, there has been difficulty with this approach when trying to apply it to unperturbed fractional differential systems $[2,6,8]$ and associated perturbed fractional differential systems with an initial time difference. The difficulty arises because there is a significant difference between initial time difference (ITD) stability [7, 9$16]$ and the classical notion of stability for fractional differential systems $[2,6]$. The classical 
notions of stability $[2-6,8,17]$ are with respect to the null solution, but ITD stability $[7,9-16]$ is with respect to the unperturbed fractional-order differential system where the perturbed fractional-order differential system and the unperturbed fractional-order differential system differ both in initial position and initial time [7, 9-16].

In this paper, we have dissipated this complexity and have a new comparison result which again gives the null solution a central role in the comparison fractionalorder differential system. This result creates many paths for continuing research by direct application and generalization $[13,15,16]$.

In Section 2, we present basic definitions and necessary rudimentary material. In Section 3, we discuss and compare the differences between the classical notion of stability and the recent notion of initial time difference (ITD) stability by means of fractionalorder differential systems. In Section 4, we have a comparison result in which the stability properties of the null solution of the comparison system imply the corresponding stability properties of the unperturbed fractional-order differential system. In Section 5, we have an other comparison result in which the stability properties of the null solution of the comparison system imply the corresponding (ITD) stability properties of the perturbed fractional-order differential system with respect to the unperturbed fractionalorder differential system.

\section{Preliminaries}

In this section we give relation among the fractional-order derivatives: Caputo, ReimannLiouville and Grünwald-Letnikov fractional-order derivatives and necessary definition of initial value problems of fractional-order differential equations with these sense.

\subsection{Fractional-Order Derivatives: Caputo, Reimann-Liouville and Grünwald-Letnikov}

Caputo's and Reimann-Liouville's definitions of fractional derivatives, are namely,

$$
\begin{aligned}
{ }^{c} D^{q} x & =\frac{1}{\Gamma(1-q)} \int_{\tau_{0}}^{t}(t-s)^{-q} x^{\prime}(s) d s, \quad \tau_{0} \leq t \leq T, \\
D^{q} x & =\frac{1}{\Gamma(p)}\left(\frac{d}{d t} \int_{\tau_{0}}^{t}(t-s)^{p-1} x(s) d s\right), \quad \tau_{0} \leq t \leq T,
\end{aligned}
$$

respectively, order of $0<q<1$ and $p+q=1$, where $\Gamma$ denotes the Gamma function.

The most important advantage for fractional-order differential equations with Caputo's derivative is the initial conditions that are the same form as that of ordinary differential equations with integer derivatives. Another difference is that the Caputo derivative for a constant $C$ is zero, while the Riemann-Liouville fractional derivative for a constant $C$ is not zero but equals to $D^{q} C=C\left(t-\tau_{0}\right)^{-q} / \Gamma(1-q)$. By using (2.1), therefore,

$$
\begin{aligned}
& { }^{c} D^{q} x(t)=D^{q}\left[x(t)-x\left(\tau_{0}\right)\right], \\
& { }^{c} D^{q} x(t)=D^{q} x(t)-\frac{x\left(\tau_{0}\right)}{\Gamma(1-q)}\left(t-\tau_{0}\right)^{-q} .
\end{aligned}
$$


In particular, if $x\left(\tau_{0}\right)=0$, we obtain

$$
{ }^{c} D^{q} x(t)=D^{q} x(t)
$$

Hence, we can see that Caputo's derivative is defined for functions for which RiemannLiouville fractional-order derivative exists.

Let us write that Grünwald-Letnikov's notion of fractional-order derivative in a convenient form

$$
D_{0}^{q} x(t)=\lim \lim _{\substack{h \rightarrow 0 \\ n h=t-\tau_{0}}} \frac{1}{h^{q}}[x(t)-S(x, h, r, q)]
$$

where $S(x, h, r, q)=\sum_{r=1}^{n}(-1)^{r+1}\left(\begin{array}{l}q \\ r\end{array}\right) x(t-r h)$. If we know that $x(t)$ is continuous and $d x(t) / d t$ exist and integrable, then Riemann-Liouville and Grünwald-Letnikov fractionalorder derivatives are connected by the relation

$$
D^{q} x(t)=D_{0}^{q} x(t)=\frac{x\left(\tau_{0}\right)\left(t-\tau_{0}\right)^{-q}}{\Gamma(1-q)}+\int_{\tau_{0}}^{t} \frac{(t-s)^{-q}}{\Gamma(1-q)} \frac{d}{d s} x(s) d s
$$

Using (2.3) implies that we have the following relations among the Caputo, RiemannLiouville and Grünwald-Letnikov fractional derivatives

$$
{ }^{c} D^{q} x(t)=D^{q}\left[x(t)-x\left(\tau_{0}\right)\right]=D_{0}^{q}\left[x(t)-x\left(\tau_{0}\right)\right]=\frac{1}{\Gamma(1-q)} \int_{\tau_{0}}^{t}(t-s)^{-q} \frac{d x(s)}{d s} d s
$$

The foregoing equivalent expressions are very useful in the study of qualitative properties of solutions of fractional differential equations.

\subsection{Existence of Euler Solution}

We consider the initial value problem of the fractional-order differential equation with Reimann-Liouville's derivative

$$
D^{q} x=f(t, x),\left.\quad x(t)\left(t-t_{0}\right)^{1-q}\right|_{t=t_{0}}=x^{0} \quad \text { for } t \geq t_{0}, t_{0} \in \mathbb{R}_{+},
$$

where $f$ is any function from $\left[t_{0}, T\right] \times \mathbb{R}^{n} \rightarrow \mathbb{R}^{n}$. Let

$$
\pi=\left[t_{0}, t_{1}, \ldots, t_{N}\right]
$$

be a partition of $\left[t_{0}, T\right]$. 
Consider the interval $\left[t_{0}, t_{1}\right]$ and observe that the right hand side of the initial value problem of fractional-order differential equation with Reimann-Liouville's derivative

$$
D^{q} x=f\left(t_{0}, x^{0}\right),\left.\quad x(t)\left(t-t_{0}\right)^{1-q}\right|_{t=t_{0}}=x^{0} \quad \text { for } t \geq t_{0}
$$

on $\left[t_{0}, t_{1}\right]$ is constant.

Therefore, the initial value problem has a unique solution of (2.10) of the fractionalorder differential equation with Reimann-Liouville's derivative given by

$$
x(t)=\frac{x^{0}\left(t-t_{0}\right)^{q-1}}{\Gamma(q)}+f\left(t_{0}, x^{0}\right) \frac{\left(t-t_{0}\right)^{q}}{\Gamma(1+q)}, \quad t \in\left[t_{0}, t_{1}\right] .
$$

Define the node $x_{1}=x\left(t_{1}\right)$ and iterate next by considering on $\left[t_{1}, t_{2}\right]$ the initial value problem

$$
D^{q} x=f\left(t_{1}, x_{1}\right),\left.\quad x_{1}(t)\left(t-t_{1}\right)^{1-q}\right|_{t=t_{1}}=x_{1}^{0} \quad \text { for } t \geq t_{1} .
$$

The next node is $x_{2}=x\left(t_{2}\right)$ and we proceed this way till an $\operatorname{arc} x_{\pi}=x_{\pi}(t)$ has been defined on all $\left[t_{0}, T\right]$. Let us employ the notation $x_{\pi}$ to emphasize the role played by the particular partition $\pi$ in determining $x_{\pi}$ which is the Euler curved arc corresponding to the partition $\pi$. The diameter of the partition $\pi$ is given by

$$
\mu_{\pi}=\max \left[t_{i}-t_{i-1}: 1 \leq i \leq N\right]
$$

Definition 2.1. An Euler solution is any curved $\operatorname{arc} x=x(t)$ which is the uniform limit of Euler curved $\operatorname{arcs} x_{\pi}$, corresponding to some sequence $\pi_{j}$ such that $\pi_{j} \rightarrow 0$, which means the convergence of the diameter $\mu_{\pi_{j}} \rightarrow 0$ as $j \rightarrow \infty$.

Now, we can give the following result on existence of an Euler solution of the initial value problem of fractional-order differential equation with Reimann-Liouville's derivative for (2.8).

Theorem 2.2. Assume that

(i) $\|f(t, x)\| \leq g(t,\|x\|),(t, x) \in\left[t_{0}, T\right] \times \mathbb{R}^{n}$, where $g \in C\left[\left[t_{0}, T\right] \times \mathbb{R}_{+}, \mathbb{R}_{+}\right], g(t, u)$ is nondecreasing in $(t, u)$;

(ii) The maximal solution $r(t)=r\left(t, t_{0}, u^{0}\right)$ of the fractional-order scalar differential equation with Reimann-Liouville's derivative

$$
D^{q} u=g(t, u),\left.u(t)\left(t-t_{0}\right)^{1-q}\right|_{t=t_{0}}=u^{0} \geq 0 \quad \text { for } t \geq t_{0}, t_{0} \in \mathbb{R}_{+}
$$

exists on $\left[t_{0}, T\right]$.

Then

(a) there exists at least one Euler solution $x(t)=x\left(t, t_{0}, x^{0}\right)$ to the initial value problem (2.8), which satisfies a Hölder condition; 
(b) any Euler solution $x(t)$ of (2.8) satisfies the relation

$$
\left\|x(t)-x^{0}(t)\right\| \leq r\left(t, t_{0}, u^{0}\right)-u^{0}, \quad t \in\left[t_{0}, T\right],
$$

where $u^{0}=\left\|x^{0}\right\|$ and $x^{0}(t)=x^{0}\left(t-t_{0}\right)^{q-1} / \Gamma(q)$.

For proof of Theorem 2.2, please see in [6].

If $f(t, x)$ in (2.8) is assumed to be continuous, then $x(t)=x\left(t, t_{0}, x^{0}\right)$, an Euler solution, is actually a solution of the initial value problem (2.8).

Theorem 2.3. Under the assumptions of Theorem 2.2 and if we suppose that $f \in C\left[\left[t_{0}, t_{0}+T\right] \times\right.$ $\left.\mathbb{R}^{n}, \mathbb{R}^{n}\right]$, then $x(t)$ is a solution of initial value problem (2.8).

For proof Theorem 2.3, please see in [6].

\subsection{Fractional-Order Differential Equations with Caputo's Derivative}

Consider the initial value problems of the fractional-order differential equations with Caputo's derivative

$$
\begin{array}{ll}
{ }^{c} D^{q} x=f(t, x), & x\left(t_{0}\right)=x_{0} \quad \text { for } t \geq t_{0}, t_{0} \in \mathbb{R}_{+}, \\
{ }^{c} D^{q} x=f(t, x), & x\left(\tau_{0}\right)=y_{0} \quad \text { for } t \geq \tau_{0}, \tau_{0} \in \mathbb{R}_{+},
\end{array}
$$

where $x_{0}=\lim _{t \rightarrow t_{0}} D^{q-1} x(t)$ and $y_{0}=\lim _{t \rightarrow \tau_{0}} D^{q-1} x(t)$ exist, and the perturbed system of initial value problem of the fractional-order differential equation with Caputo's derivative of (2.17)

$$
{ }^{c} D^{q} y=F(t, y), \quad y\left(\tau_{0}\right)=y_{0} \quad \text { for } t \geq \tau_{0} \geq t_{0}
$$

where $y_{0}=\lim _{t \rightarrow \tau_{0}} D^{q-1} y(t)$, exists, and $f, F \in C\left[\left[t_{0}, \tau_{0}+T\right] \times \mathbb{R}^{n}, \mathbb{R}^{n}\right]$; satisfy a local Lipschitz condition on the set $\mathbb{R}_{+} \times S \rho, S \rho=\left[x \in \mathbb{R}^{n}:\|x\|<\rho<\infty\right]$ and $f(t, 0)=0$ for $t \geq 0$. In particular, $F(t, y)=f(t, y)+R(t, y)$, we have a special case of $(2.18)$ and $R(t, y)$ is said to be perturbation term.

Corollary 2.4. Let $0<q<1$, and $f:\left(t_{0}, t_{0}+T\right] \times S \rho \rightarrow \mathbb{R}$ a function such that $f(t, x) \in L\left(t_{0}, t_{0}+T\right)$ for any $x \in S \rho$. If $x(t) \in L\left(t_{0}, t_{0}+T\right)$, then $x(t)$ satisfies a.e. the initial value problems of the fractionalorder differential equations with Reimann-Liouville's derivative (2.19) if, and only if, $x(t)$ satisfies a.e. the Volterra fractional-order integral equation (2.20).

For proof of Corollary 2.4, please see in [2].

We assume that we have sufficient conditions to the existence and uniqueness of solutions through $\left(t_{0}, x_{0}\right)$ and $\left(\tau_{0}, y_{0}\right)$. If $f \in C\left[\left[t_{0}, t_{0}+T\right] \times \mathbb{R}^{n}, \mathbb{R}^{n}\right]$ and $x(t)$ is the solution of

$$
D^{q} x=f(t, x),\left.\quad x(t)\left(t-t_{0}\right)^{1-q}\right|_{t=t_{0}}=x^{0} \quad \text { for } t \geq t_{0}, t_{0} \in \mathbb{R}_{+},
$$


where $D^{q} x$ is the Reimann-Liouville fractional-order derivative of $x$ as in (2.2), then it also satisfies the Volterra fractional-order integral equation

$$
x(t)=\frac{x^{0}\left(t-t_{0}\right)^{q-1}}{\Gamma(q)}+\frac{1}{\Gamma(q)} \int_{t_{0}}^{t}(t-s)^{q-1} f(s, x(s)) d s, \quad t_{0} \leq t \leq t_{0}+T
$$

that is, every solution of (2.20) is also a solution of (2.19); for detail please see [2].

We will only give the basic existence and uniqueness result with the Lipschitz condition by using contraction mapping theorem and a weighted norm with Mittag-Leffler function in [6].

Theorem 2.5. Assume that

(i) $f \in C\left[R, \mathbb{R}^{n}\right]$ and bounded by $M$ on $R$, where $R=\left[(t, x): t_{0} \leq t \leq t_{0}+T,\left\|x-x_{0}\right\| \leq b\right]$;

(ii) $\|f(t, x)-f(t, y)\| \leq L\|x-y\|, L>0,(t, x) \in R$, where the inequalities are componentwise.

Then there exists a unique solution $x(t)=x\left(t, t_{0}, x_{0}\right)$ on $\left[t_{0}, t_{0}+\alpha\right]$ for the initial value problem of the fractional-order differential equation with Caputo's derivative of (2.16), where $\alpha=$ $\min \left[T,(b \Gamma(q+1) / M)^{1 / q}\right]$.

For proof of Theorem 2.5, please see in [6].

\subsection{Stability Criteria with ITD and Lyapunov-Like Function}

Before we can establish our comparison theorem and Lyapunov stability criteria for initial time difference, we need to introduce the following definitions of ITD stability and Lyapunovlike functions.

Definition 2.6. The solution $y\left(t, \tau_{0}, y_{0}\right)$ of the initial value problems of fractional-order differential equation with Caputo's derivative of $(2.18)$ through $\left(\tau_{0}, y_{0}\right)$ is said to be initial time difference stable with respect to the solution $\tilde{x}\left(t, \tau_{0}, x_{0}\right)=x\left(t-\eta, t_{0}, x_{0}\right)$, where $x\left(t, t_{0}, x_{0}\right)$ is any solution of the initial value problems of fractional-order differential equation with Caputo's derivative of (2.16) for $t \geq \tau_{0}, \tau_{0} \in \mathbb{R}_{+}$and $\eta=\tau_{0}-t_{0}$ if and only if given any $\epsilon>0$ there exist $\delta_{1}=\delta_{1}\left(\epsilon, \tau_{0}\right)>0$ and $\delta_{2}=\delta_{2}\left(\epsilon, \tau_{0}\right)>0$, such that

$$
\left\|y\left(t, \tau_{0}, y_{0}\right)-x\left(t-\eta, t_{0}, x_{0}\right)\right\|<\epsilon, \quad \text { whenever }\left\|y_{0}-x_{0}\right\|<\delta_{1},\left|\tau_{0}-t_{0}\right|<\delta_{2} \text { for } t \geq \tau_{0}
$$

If $\delta_{1}, \delta_{2}$ are independent of $\tau_{0}$, then the solution $y\left(t, \tau_{0}, y_{0}\right)$ of the initial value problems of fractional-order differential equation with Caputo's derivative of (2.18) is initial time difference uniformly stable with respect to the fractional solution $x\left(t-\eta, t_{0}, x_{0}\right)$. If the solution of initial value problems of fractional-order differential equation with Caputo's derivative of $y\left(t, \tau_{0}, y_{0}\right)$ of the fractional system $(2.18)$ through $\left(\tau_{0}, y_{0}\right)$ is initial time difference stable and there exist $\gamma_{1}\left(\tau_{0}\right)>0$ and $\gamma_{2}\left(\tau_{0}\right)>0$ such that

$$
\lim _{t \rightarrow \infty}\left\|y\left(t, \tau_{0}, y_{0}\right)-x\left(t-\eta, t_{0}, x_{0}\right)\right\|=0
$$


for all $y\left(t, \tau_{0}, y_{0}\right)$ and $x\left(t-\eta, t_{0}, x_{0}\right)$ with $\left\|y_{0}-x_{0}\right\|<\gamma_{1}$ and $\left|\tau_{0}-t_{0}\right|<\gamma_{2}$ for $t \geq \tau_{0}$, then it is said to be initial time difference asymptotically stable with respect to the fractional solution $x\left(t-\eta, t_{0}, x_{0}\right)$. It is initial time difference uniformly asymptotically stable with respect to the fractional solution $x\left(t-\eta, t_{0}, x_{0}\right)$ if $\gamma_{1}$ and $\gamma_{2}$ are independent of $\tau_{0}$.

Definition 2.7. A function $\phi(r)$ is said to belong to the class $\mathcal{K}$ if $\phi \in C\left[(0, \rho), \mathbb{R}_{+}\right], \phi(0)=0$, and $\phi(r)$ is strictly monotone increasing in $r$.

Definition 2.8. For any Lyapunov-like function $V(t, x) \in C\left[\mathbb{R}_{+} \times \mathbb{R}^{n}, \mathbb{R}_{+}\right]$, we define the fractional-order Dini derivatives in Caputo's sense ${ }^{c} D_{+}^{q} V(t, x)$ and ${ }^{c} D_{-}^{q} V(t, x)$ as follows

$$
\begin{aligned}
& { }^{c} D_{+}^{q} V(t, x)=\lim _{h \rightarrow 0^{+}} \sup \frac{1}{h^{q}}\left[V(t, x)-V\left(t-h, x-h^{q} f(t, x)\right)\right], \\
& { }^{c} D_{-}^{q} V(t, x)=\lim _{h \rightarrow 0^{-}} \inf \frac{1}{h^{q}}\left[V(t, x)-V\left(t-h, x-h^{q} f(t, x)\right)\right]
\end{aligned}
$$

for $(t, x) \in \mathbb{R}_{+} \times \mathbb{R}^{n}$.

Definition 2.9. For a real-valued function $V(t, x) \in C\left[\mathbb{R}_{+} \times \mathbb{R}^{n}, \mathbb{R}_{+}\right]$, we define the generalized fractional-order derivatives (Dini-like derivatives) in Caputo's sense ${ }_{*}^{c} D_{+}^{q} V(t, y-\tilde{x})$ and ${ }_{*}^{c} D_{-}^{q} V(t, y-\tilde{x})$ as follows

$$
\begin{aligned}
& { }_{*}^{c} D_{+}^{q} V(t, y-\tilde{x})=\lim _{h \rightarrow 0^{+}} \sup \frac{1}{h^{q}}\left[V(t, y-\tilde{x})-V\left(t-h, y-\tilde{x}-h^{q}(F(t, y)-\tilde{f}(t, \tilde{x}))\right)\right], \\
& { }_{*}^{c} D_{-}^{q} V(t, y-\tilde{x})=\lim _{h \rightarrow 0^{-}} \inf \frac{1}{h^{q}}\left[V(t, y-\tilde{x})-V\left(t-h, y-\tilde{x}-h^{q}(F(t, y)-\tilde{f}(t, \tilde{x}))\right)\right]
\end{aligned}
$$

for $(t, x) \in \mathbb{R}_{+} \times \mathbb{R}^{n}$.

\section{Comparing Fractional Stability with Fractional (ITD) Stability}

\subsection{Fractional Classical Notion of Stability}

Let $x\left(t, t_{0}, x_{0}\right)$ and $x\left(t, t_{0}, y_{0}\right)$ be any solutions of the initial value problems of fractional-order differential equations with Caputo's derivative of (2.16) and of (3.1), respectively,

$$
{ }^{c} D^{q} x=f(t, x), \quad x\left(t_{0}\right)=y_{0} \quad \text { for } t \geq t_{0}, t_{0} \in \mathbb{R}_{+},
$$

where $f \in C\left[\left[t_{0}, T\right] \times \mathbb{R}^{n}, \mathbb{R}^{n}\right]$.

Assume that $f(t, 0)=0, t \in \mathbb{R}_{+}$so that $x=0$ is a null solution of fractional-order differential equation with Caputo's derivative of $(3.1)$ through $\left(t_{0}, 0\right)$. Now, we can state the well-known definitions concerning the stability of the null solution. 
Definition 3.1. The null solution $x=0$ of fractional-order differential equation with Caputo's derivative of (3.1) is said to be stable if and only if for each $\epsilon>0$ and for all $t_{0} \in \mathbb{R}_{+}$, there exists a positive function $\delta=\delta\left(\epsilon, t_{0}\right)$ that is continuous in $t_{0}$ for each $\epsilon$ such that

$$
\left\|x_{0}\right\| \leq \delta \text { implies }\left\|x\left(t, t_{0}, x_{0}\right)\right\|<\epsilon \text { for } t \geq t_{0} .
$$

If $\delta$ is independent of $t_{0}$, then the null solution $x=0$ of initial value problems of fractionalorder differential equation with Caputo's derivative of (3.1) is said to be uniformly stable.

Definition 3.2. The solution $x\left(t, t_{0}, y_{0}\right)$ of initial value problems of fractional-order differential equation with Caputo's derivative of (3.1) through $\left(t_{0}, y_{0}\right)$ is said to be stable with respect to the solution $x\left(t, t_{0}, x_{0}\right)$ of fractional-order differential equation with Caputo's derivative of (3.1) for $t \geq t_{0} \in \mathbb{R}_{+}$if and only if given any $\epsilon>0$ there exists a positive function $\delta=\delta\left(\epsilon, t_{0}\right)$ that is continuous in $t_{0}$ for each $\epsilon$ such that

$$
\left\|y_{0}-x_{0}\right\| \leq \delta \text { implies }\left\|x\left(t, t_{0}, y_{0}\right)-x\left(t, t_{0}, x_{0}\right)\right\|<\epsilon \quad \text { for } t \geq t_{0}
$$

If $\delta$ is independent of $t_{0}$, then the solution of the fractional-order differential equation with Caputo's derivative of (3.1) is uniformly stable with respect to the solution $x\left(t, t_{0}, x_{0}\right)$ of (2.16).

We remark that for the purpose of studying the classical stability of a given solution $x\left(t, t_{0}, y_{0}\right)$ of the initial value problem of fractional-order differential equation with Caputo's derivative of (3.1), it is convenient to make a change of variable. Let $x\left(t, t_{0}, x_{0}\right)$ and $x\left(t, t_{0}, y_{0}\right)$ be the unique solutions of the fractional-order differential equations with Caputo's derivative (2.16) and (3.1), respectively, and set $z\left(t, t_{0}, y_{0}-x_{0}\right)=x\left(t, t_{0}, y_{0}\right)-x\left(t, t_{0}, x_{0}\right)$ for $t \geq t_{0}$. Then

$$
\begin{aligned}
& { }^{c} D^{q} z\left(t, t_{0}, y_{0}-x_{0}\right)={ }^{c} D^{q} x\left(t, t_{0}, y_{0}\right)-{ }^{c} D^{q} x\left(t, t_{0}, x_{0}\right), \\
& { }^{c} D^{q} z\left(t, t_{0}, y_{0}-x_{0}\right)=f\left(t, z\left(t, t_{0}, y_{0}-x_{0}\right)+x\left(t, t_{0}, x_{0}\right)\right)-f\left(t, x\left(t, t_{0}, x_{0}\right)\right), \\
& { }^{c} D^{q} z\left(t, t_{0}, y_{0}-x_{0}\right)=\tilde{f}\left(t, z\left(t, t_{0}, y_{0}-x_{0}\right)\right) .
\end{aligned}
$$

It is easy to observe that $z\left(t, t_{0}, y_{0}-x_{0}\right) \equiv 0$ is a solution of the transformed initial value problems of the fractional differential equation with Caputo's derivative if $y_{0}-x_{0}=0$ which implies $f(t, 0)=0$. Since ${ }^{c} D^{q} z(t)=0$ and $z\left(t, t_{0}, 0\right)=0$ is the null solution, the solution of $x\left(t, t_{0}, x_{0}\right)$ initial value problems of the fractional-order differential equation with Caputo's derivative of (2.16) corresponds to the identically null solution of ${ }^{c} D^{q} z=\tilde{f}(t, z)$, where $\tilde{f}\left(t, z\left(t, t_{0}, y_{0}-x_{0}\right)\right)=f\left(t, z\left(t, t_{0}, y_{0}-x_{0}\right)+x\left(t, t_{0}, x_{0}\right)\right)-f\left(t, x\left(t, t_{0}, x_{0}\right)\right)$. Hence, we can always assume, without any loss of generality, that $x\left(t, t_{0}, x_{0}\right) \equiv 0$ is the null solution of the given fractional-order differential equation with Caputo's derivative of (3.1) and we can limit our study of stability to that of the null solution $[2,6,8]$. However, it is impossible to do the same transformation for fractional (ITD) stability which we deal with it. 


\subsection{New Notion of Fractional (ITD) Stability}

Let $x\left(t, \tau_{0}, y_{0}\right)$ be a fractional solution of (2.17) and $\tilde{x}\left(t, \tau_{0}, x_{0}\right)=x\left(t-\eta, t_{0}, x_{0}\right)$, where $x\left(t, t_{0}, x_{0}\right)$ is any solution of initial value problems of the fractional-order differential equations with Caputo's derivative of the system (2.16) for $t \geq \tau_{0} \geq 0$. Let us make a transformation similar to that in (3.5). Set $z\left(t, \tau_{0}, y_{0}-x_{0}\right)=x\left(t, \tau_{0}, y_{0}\right)-x\left(t-\eta, t_{0}, x_{0}\right)$ for $t \geq \tau_{0}$. Then

$$
\begin{aligned}
& { }^{c} D^{q} z\left(t, \tau_{0}, y_{0}-x_{0}\right)={ }^{c} D^{q} x\left(t, \tau_{0}, y_{0}\right)-{ }^{c} D^{q} x\left(t-\eta, t_{0}, x_{0}\right), \\
& { }^{c} D^{q} z\left(t, \tau_{0}, y_{0}-x_{0}\right)=\tilde{f}\left(\eta ; t, z\left(t, \tau_{0}, y_{0}-x_{0}\right)\right) .
\end{aligned}
$$

One can observe that even if $y_{0}=x_{0}, z\left(t, \tau_{0}, 0\right)$ is not zero and is not the null solution of the initial value problems of transformed fractional-order differential equation with Caputo's derivative and the solution. $x\left(t-\eta, t_{0}, x_{0}\right)$ does not correspond to the identically zero solution of ${ }^{c} D^{q} z=\tilde{f}(\eta ; t, z)$. Therefore, we cannot use stability properties of the fractional-order differential equation with Caputo's derivative of null solution in order to find fractional (ITD) stability properties using this approach.

\section{A Fractional Comparison Result}

In our earlier work and in the work of others [4-6], the differences between the classical notion of fractional stability and fractional ITD stability did not allow the use of the behavior of the null solution in our fractional ITD stability analysis. The main result presented in this section resolves those difficulties with a new approach that allows the use of the fractional stability of the null solution of the comparison system to predict the Caputo's fractional stability properties of $y\left(t, \tau_{0}, y_{0}\right)$ the solution of fractional-order differential equation with Caputo's derivative of (2.18) with respect to $\tilde{x}(t)=x\left(t-\eta, t_{0}, x_{0}\right)$, where $x\left(t, t_{0}, x_{0}\right)$ is any solution of the fractional-order differential equation with Caputo's derivative of (2.16).

Let $0<q<1$ and $p+q=1$. The function space is denoted by $C_{p}\left[\left[\tau_{0}, T\right], \mathbb{R}\right]$ as follows:

$$
C_{p}\left[\left[\tau_{0}, T\right], \mathbb{R}\right]=\left[u \in C\left[\left(\tau_{0}, T\right], \mathbb{R}\right],\left(t-\tau_{0}\right)^{1-q} u(t) \in C\left[\left[\tau_{0}, T\right], \mathbb{R}\right]\right]
$$

The Riemann-Liouville fractional derivative is defined by

$$
D^{q} x(t)=\frac{x\left(\tau_{0}\right)\left(t-\tau_{0}\right)^{-q}}{\Gamma(1-q)}+\int_{\tau_{0}}^{t} \frac{(t-s)^{-q}}{\Gamma(1-q)} \frac{d}{d s} x(s) d s
$$

Now, we will prove the following comparison result.

Theorem 4.1. Assume that $m \in C_{p}\left[\left[\tau_{0}, T\right], \mathbb{R}_{+}\right]$is locally Hölder continuous, $g \in C_{p}\left[\left[\tau_{0}, T\right] \times\right.$ $\left.\mathbb{R}_{+}, \mathbb{R}\right]$ and

$$
D^{q} m(t) \leq g(t, m(t)), \quad \tau_{0} \leq t \leq T .
$$


Let $r(t)$ be the maximal solution of the initial value problem of fractional-order scalar differential equation with Riemann-Liouville's derivative

$$
D^{q} u(t)=g(t, u(t)),\left.\quad u(t)\left(t-\tau_{0}\right)^{1-q}\right|_{t=\tau_{0}}=u^{0} \geq 0
$$

existing on $\left[\tau_{0}, T\right]$ such that $m^{0} \leq u^{0}$, where $m^{0}=\left.m(t)\left(t-\tau_{0}\right)^{1-q}\right|_{t=\tau_{0}}$. Then we have

$$
m(t) \leq r(t), \quad \tau_{0} \leq t \leq T .
$$

Proof. In view of the definition of the maximal solution $r(t)$ of the fractional-order differential equation with Riemann-Liouville's derivative of (4.4), it is enough to prove that

$$
m(t)<u(t, \epsilon), \quad \tau_{0} \leq t \leq T,
$$

where $u(t, \epsilon)$ is any solution of the initial value problem of fractional-order scalar differential equation with Riemann-Liouville's derivative

$$
D^{q} u=g(t, u)+\epsilon \text { with initial value } u^{0}+\epsilon, \quad \epsilon>0 .
$$

Now it follows from (4.7) that

$$
D^{q} u(t, \epsilon)>g(t, u(t, \epsilon))
$$

Then by applying the comparison result [2, Theorem 2.1 in page 23], we get (4.6) and since $\lim _{\epsilon \rightarrow 0} u(t, \epsilon)=r(t)$ uniformly on each compact set $\tau_{0} \leq t \leq T_{0}<T$. Hence,

$$
m(t) \leq \lim _{\epsilon \rightarrow 0} u(t, \epsilon)=r(t), \quad \tau_{0} \leq t \leq T .
$$

The proof is complete.

Theorem 4.2. Assume that $f \in C\left[\left[\tau_{0}, T\right] \times S(\rho), \mathbb{R}^{n}\right]$ and $[x, f(t, x)]_{+} \leq g(t,\|x\|],(t, x) \in$ $\left[\tau_{0}, T\right] \times S(\rho)$, where $[x, f(t, x)]_{+}=\lim _{h \rightarrow 0^{+}} \sup \left(1 / h^{q}\right)\left[\|x\|-\left\|x-h^{q} f(t, x)\right\|\right]$ and $g \in C\left[\left[\tau_{0}, T\right] \times\right.$ $\left.\mathbb{R}_{+}, \mathbb{R}\right], g(t, 0) \equiv 0$. Then the stability properties of the trivial solution of the comparison initial value problem of fractional-order differential equation with Caputo's derivative

$$
{ }^{c} D^{q} u=g(t, u(t)), \quad u\left(\tau_{0}\right)=u_{0} \geq 0
$$

imply the corresponding stability results of the solution $x\left(t, \tau_{0}, y_{0}\right)$ of the initial value problem of the fractional-order differential equation with Caputo's derivative of (2.17), respectively.

Proof. Let $u \equiv 0$ of (4.10) be stable. Then given $0<\epsilon<\rho$ and $\tau_{0} \geq 0$, there exists for a $\delta=\delta\left(\epsilon, \tau_{0}\right)>0$ with the property that

$$
0 \leq u_{0}<\delta \text { implies } u\left(t, \tau_{0}, u_{0}\right)<\epsilon, \quad t \geq \tau_{0} .
$$


We claim that the trivial solution of (2.17) is stable for these $\epsilon$ and $\delta$. If this was false, then there would exists a solution $x(t)$ of (2.17) and $t_{1}>\tau_{0}$ such that

$$
\left\|x\left(t_{1}\right)\right\|=\epsilon, \quad\|x(t)\| \leq \epsilon \quad \text { for } \tau_{0} \leq t \leq t_{1} .
$$

For $\left[\tau_{0}, t_{1}\right]$, we set $m(t)=\|x(t)\|$ and choose $\left\|y_{0}\right\| \leq u_{0}$. Then we have

$$
x(t)-S(x, h, r, q)=h^{q} f(t, x(t))+\epsilon\left(h^{q}\right)
$$

which shows that $S(x, h, r, q)=x(t)-h^{q} f(t, x(t))-\epsilon\left(h^{q}\right)$ where $\epsilon\left(h^{q}\right) / h^{q} \rightarrow 0$ as $h \rightarrow 0^{+}$. Now, by using the fractional-order Dini derivatives in Caputo's sense ${ }^{c} D_{+}^{q} m(t)$ in Definition 2.8 as in (2.23), we have

$$
{ }^{c} D_{+}^{q} m(t)=\lim _{h \rightarrow 0^{+}} \sup \frac{1}{h^{q}}\left[\|x(t)\|-\left\|x(t)-h^{q} f(t, x(t))\right\|\right] \leq g(t, m(t))
$$

for $\tau_{0} \leq t \leq t_{1}$ and $m\left(\tau_{0}\right)=\left\|y_{0}\right\|$.

This yields by comparison Theorem 4.1, the estimate

$$
\left\|x\left(t, \tau_{0}, y_{0}\right)\right\| \leq r\left(t, \tau_{0},\left\|y_{0}\right\|\right), \quad \tau_{0} \leq t \leq t_{1}
$$

where $r\left(t, \tau_{0}, u_{0}\right)$ is the maximal solution of (4.4). At $t=t_{1}$, we therefore arrive at the following contradiction

$$
\epsilon=\left\|x\left(t_{1}, \tau_{0}, y_{0}\right)\right\| \leq r\left(t_{1}, \tau_{0},\left\|y_{0}\right\|\right)<\epsilon
$$

Therefore, this do justify our claim. Hence the trivial solution of initial value problem of the fractional-order differential equation with Caputo's derivative of (2.17) is stable.

Next suppose that $u \equiv 0$ is asymptotically stable. Since this implies by definition of the stability of $u \equiv 0$, the stability of the trivial solution of (4.10) is the foregoing argument. This means the inequality (4.15) holds for all $\tau_{0} \leq t$ and hence, it is clear, by hypothesis, that if $\left\|x_{0}\right\|<\delta_{0}$, then $\lim _{t \rightarrow \infty}\left\|x\left(t, \tau_{0}, x_{0}\right)\right\|=0$. The proof is therefore complete.

\section{An Initial Time Difference Fractional Comparison Result}

In this section, we have an other comparison result in which the stability properties of the null solution of the comparison system imply the corresponding initial time difference stability properties of the perturbed fractional-order differential system in Caputo's sense with respect to the unperturbed fractional-order differential system in Caputo's sense.

Theorem 5.1. Let $f, F \in C\left[\left[t_{0}, T\right] \times \mathbb{R}^{n}, \mathbb{R}^{n}\right]$, and let

$$
G(t, r)=\max _{\tilde{x}, y \in \bar{B}\left(x_{0} ; r\right)}\|F(t, y)-\tilde{f}(t, \tilde{x})\|,
$$


where $G(t, r) \in C\left[\mathbb{R}_{+} \times \mathbb{R}_{+}, \mathbb{R}_{+}\right]$and $\bar{B}$ is closed ball with center at $x_{0}$ and radius $r$. Assume that $r^{*}\left(t, \tau_{0},\left\|y_{0}-x_{0}\right\|\right)$ is the maximal solution of initial value problem of fractional-order differential equation with Caputo's derivative ${ }^{c} D^{q} u=G(t, u), u\left(\tau_{0}\right)=\left\|y_{0}-x_{0}\right\|$ through $\left(\tau_{0},\left\|y_{0}-x_{0}\right\|\right)$. $\tilde{x}\left(t, \tau_{0}, x_{0}\right)=x\left(t-\eta, t_{0}, x_{0}\right)$, where $x\left(t, t_{0}, x_{0}\right)$ is any solution of fractional-order differential equation of (2.16) for $t \geq \tau_{0} \geq 0, t_{0} \in \mathbb{R}_{+}$, and $\eta=\tau_{0}-t_{0}$, and $y\left(t, \tau_{0}, y_{0}\right)$ is the solution of fractional-order differential equation (2.18) with Caputo's derivatives. Then

$$
\left\|y\left(t, \tau_{0}, y_{0}\right)-x\left(t-\eta, t_{0}, x_{0}\right)\right\| \leq r^{*}\left(t, \tau_{0},\left\|y_{0}-x_{0}\right\|\right) \quad \text { for } t \geq \tau_{0}
$$

Proof. Let $m(t)=\left\|y\left(t, \tau_{0}, y_{0}\right)-\tilde{x}\left(t, \tau_{0}, x_{0}\right)\right\|$ for $t \geq \tau_{0}$. Then by using the fractional-order Dini derivatives in Caputo's sense ${ }^{c} D_{+}^{q} m(t)$ in Definition 2.8 as in (2.23), we obtain

$$
\begin{aligned}
{ }^{c} D_{+}^{q} m(t) & ={ }^{c} D_{+}^{q}\left[\left\|y\left(t, \tau_{0}, y_{0}\right)-\tilde{x}\left(t, \tau_{0}, x_{0}\right)\right\|\right] \\
& \leq\left\|F\left(t, y\left(t, \tau_{0}, y_{0}\right)\right)-\tilde{f}\left(t, \tilde{x}\left(t, \tau_{0}, x_{0}\right)\right)\right\| \\
& \leq \max _{\tilde{x}, y \in \bar{B}\left(x_{0} ; m(t)\right)}\|F(t, y)-\tilde{f}(t, \tilde{x})\| \\
& =G(t, m(t)) .
\end{aligned}
$$

Therefore, ${ }^{c} D_{+}^{q} m(t) \leq G(t, m(t)), m\left(\tau_{0}\right)=\left\|y_{0}-x_{0}\right\|$ and by using a standard result in [6], we get $m(t) \leq r^{*}\left(t, \tau_{0},\left\|y_{0}-x_{0}\right\|\right)$.

Now, we can formulate the comparison results via Lyapunov-like functions.

Theorem 5.2. Let $V(t, z) \in C\left[\mathbb{R}_{+} \times \mathbb{R}^{n}, \mathbb{R}_{+}\right]$and $V(t, z)$ be locally Lipschitzian in $z$. Assume that the generalized fractional-order derivatives (Dini-like derivatives) in Caputo's sense

$$
{ }_{*}^{c} D_{+}^{q} V(t, y-\tilde{x})=\lim _{h \rightarrow 0^{+}} \sup \frac{1}{h^{q}}\left[V(t, y-\tilde{x})-V\left(t-h, y-\tilde{x}-h^{q}(F(t, y)-\tilde{f}(t, \tilde{x}))\right)\right]
$$

satisfies ${ }_{*}^{c} D_{+}^{q} V(t, y-\tilde{x}) \leq G(t, V(t, y-\tilde{x}))$ with $(t, \tilde{x}),(t, y) \in \mathbb{R}_{+} \times \mathbb{R}^{n}$, where $G(t, u) \in C\left[\mathbb{R}_{+} \times\right.$ $\left.\mathbb{R}_{+}, \mathbb{R}\right]$. Let $r(t)=r\left(t, \tau_{0}, u_{0}\right)$ be the maximal solution of the fractional-order differential equation ${ }^{c} D^{q} u=G(t, u), u\left(\tau_{0}\right)=u_{0} \geq 0$, for $t \geq t_{0}$. If $\tilde{x}(t)=x\left(t-\eta, t_{0}, x_{0}\right)$, where $x\left(t, t_{0}, x_{0}\right)$ is any solution of the system (2.16) for $t \geq \tau_{0} \geq 0, t_{0} \in \mathbb{R}_{+}$and $\eta=\tau_{0}-t_{0}$ and $y(t)=y\left(t, \tau_{0}, y_{0}\right)$ is any solution of (2.18) for $t \geq \tau_{0}$ such that $V\left(\tau_{0}, y_{0}-x_{0}\right) \leq u_{0}$, then $V(t, y(t)-\widetilde{x}(t)) \leq r(t)$ for $t \geq \tau_{0}$.

Proof. Let $\tilde{x}(t)=x\left(t-\eta, t_{0}, x_{0}\right)$, where $x\left(t, t_{0}, x_{0}\right)$ is any solution of the system fractional-order differential equation (2.16) for $t \geq \tau_{0} \geq 0, t_{0} \in \mathbb{R}_{+}$, and $\eta=\tau_{0}-t_{0}$ and $y(t)=y\left(t, \tau_{0}, y_{0}\right)$ is any solution of fractional-order differential equation (2.18) for $t \geq \tau_{0}$ such that $V\left(\tau_{0}, y_{0}-x_{0}\right) \leq u_{0}$ holds. Define $m(t)=V(t, y(t)-\tilde{x}(t))$ for $t \geq \tau_{0}$ so that $m\left(t_{0}\right) \leq u_{0}$. Then for small enough $h>0$, we get

$$
m(t)-m(t-h)=V(t, y(t)-\tilde{x}(t))-V(t-h, y(t-h)-\tilde{x}(t-h)) .
$$


Since $V$ is locally Lipschitzian in $\tilde{x}$ and fractional-order Dini derivatives in Caputo's sense ${ }^{c} D_{+}^{q} m(t)$

$$
\begin{aligned}
V(t, y(t)-\tilde{x}(t))-V(t-h, S(y-\tilde{x}, h, r, q)) \\
\leq \\
\quad V(t, y(t)-\tilde{x}(t))-V\left(t-h,(y(t)-\tilde{x}(t))-h^{q}(F(t, y)-\tilde{f}(t, \tilde{x}))\right) \\
\quad+V\left(t-h,(y(t)-\tilde{x}(t))-h^{q}(F(t, y)-\tilde{f}(t, \tilde{x}))\right)-V(t-h, S(y-\tilde{x}, h, r, q)),
\end{aligned}
$$

where $L>0$ is the Lipschitz constant, $S(y-\tilde{x}, h, r, q)=y-\tilde{x}-h^{q}[(F(t, y)-\tilde{f}(t, \tilde{x}))]-\left[\epsilon_{1}\left(h^{q}\right)-\right.$ $\left.\left.\epsilon_{2}\left(h^{q}\right)\right)\right]$ and $\epsilon_{1}$ and $\epsilon_{2}$ are error terms

$$
\begin{aligned}
{ }^{c} D_{+}^{q} m(t) \leq & \lim _{h \rightarrow 0^{+}} \sup \frac{1}{h^{q}}\left[V(t, y(t)-\tilde{x}(t))-V\left(t-h,(y(t)-\tilde{x}(t))-h^{q}(F(t, y)-\tilde{f}(t, \tilde{x}))\right)\right] \\
& +\lim _{h \rightarrow 0^{+}} \sup \frac{1}{h^{q}}\left[V\left(t-h,(y(t)-\tilde{x}(t))-h^{q}(F(t, y)-\tilde{f}(t, \tilde{x}))\right)-V(t-h, S(y-\tilde{x}, h, r, q))\right], \\
{ }^{c} D_{+}^{q} m(t) \leq & \lim _{h \rightarrow 0^{+}} \sup \frac{1}{h^{q}}\left[L\left(\epsilon_{1}\left(h^{q}\right)-\epsilon_{2}\left(h^{q}\right)\right)\right] \\
& +\lim _{h \rightarrow 0^{+}} \sup \frac{1}{h^{q}}\left[V(t, y(t)-\tilde{x}(t))-V(t-h,(y(t)-\tilde{x}(t)))-h^{q}(F(t, y)-\tilde{f}(t, \tilde{x}))\right],
\end{aligned}
$$

where $\lim _{h \rightarrow 0^{+}} \sup L\left[\left(\epsilon_{1}\left(h^{q}\right)-\epsilon_{2}\left(h^{q}\right)\right) / h^{q}\right] \rightarrow 0$. Since ${ }_{*}^{c} D_{+}^{q} V(t, y-\tilde{x})$ is the generalized fractional-order derivatives (Dini-like derivatives) in Caputo's sense, we have

$$
\begin{gathered}
{ }^{c} D_{+}^{q} m(t) \leq{ }_{*}^{c} D_{+}^{q} V(t, y(t)-\tilde{x}(t)) \leq G(t, V(t, y(t)-\tilde{x}(t)))=G(t, V(t, m(t))), \\
{ }^{c} D_{+}^{q} m(t) \leq G(t, V(t, m(t))), \quad m\left(\tau_{0}\right)=V\left(\tau_{0}, y\left(\tau_{0}\right)-\tilde{x}\left(\tau_{0}\right)\right) \leq u_{0} .
\end{gathered}
$$

By using Theorem 5.1, this implies

$$
m(t)=V(t, y(t)-\tilde{x}(t)) \leq r\left(t, \tau_{0},\left\|y_{0}-x_{0}\right\|\right),
$$

where $r$ is the maximal solution of ${ }^{c} D^{q} u=G(t, u), u\left(\tau_{0}\right)=u_{0} \geq 0$ for $t \geq t_{0}$.

Now, we present the main comparison result that yields knowledge of initial time difference fractional stability properties if we know the stability properties of the null solution of the fractional comparison system.

Theorem 5.3. Assume that

(i) let $V(t, z) \in C\left[\mathbb{R}_{+} \times \mathbb{R}^{n}, \mathbb{R}_{+}\right]$be locally Lipschitzian in $z$, positive definite and decrescent where the fractional-order Dini derivatives in Caputo's sense ${ }^{c} D_{+}^{q} m(t)$

$$
{ }^{c} D_{+}^{q} m(t) \leq \lim _{h \rightarrow 0^{+}} \sup \frac{1}{h^{q}}\left[V(t, y(t)-\tilde{x}(t))-V\left(t-h,(y-\tilde{x})-h^{q}(F(t, y)-\tilde{f}(t, \tilde{x}))\right)\right]
$$


satisfies ${ }_{*}^{c} D_{+}^{q} V(t, y-\tilde{x}) \leq G(t, V(t, y-\tilde{x}))$ for $(t, \tilde{x})$ and $(t, y) \in \mathbb{R}_{+} \times \mathbb{R}^{n}$, where $G(t, u) \in C\left[\mathbb{R}_{+} \times\right.$ $\left.\mathbb{R}_{+}, \mathbb{R}\right]$ and the generalized fractional-order (Dini-like) derivatives in Caputo's sense ${ }_{*}^{c} D_{+}^{q} V(t, x)$,

$$
a(\|x\|) \leq V(t, x) \leq b(\|x\|), \quad(t, x) \in \mathbb{R}_{+} \times \mathbb{R}^{n}, a, b \in \mathcal{K} .
$$

(ii) Let $r(t)=r\left(t, \tau_{0}, u_{0}\right)$ be the maximal solution of the fractional-order differential equation with Caputo's derivative

$$
{ }^{c} D^{q} u=G(t, u), \quad u\left(\tau_{0}\right)=u_{0} \geq 0 \quad \text { for } t \geq \tau_{0} .
$$

Then the stability properties of the null solution of the fractional-order differential system with Caputo's derivative (5.12) with $G(t, 0)=0$ imply the corresponding stability properties of $y\left(t, \tau_{0}, y_{0}\right)$ any solution of fractional-order differential system with Caputo's derivative (2.18) with respect to $\tilde{x}\left(t, \tau_{0}, x_{0}\right)=x\left(t-\eta, t_{0}, x_{0}\right)$, where $x\left(t, t_{0}, x_{0}\right)$ is any solution of fractional-order differential system with Caputo's derivative of (2.16).

Proof. Let $\tilde{x}(t)=x\left(t-\eta, t_{0}, x_{0}\right)$, where $x\left(t, t_{0}, x_{0}\right)$ is any solution of the fractional-order differential system with Caputo's derivative of (2.16) for $t \geq t_{0} \geq 0, t_{0} \in \mathbb{R}_{+}$, and $\eta=\tau_{0}-t_{0}$ and $y(t)=y\left(t, \tau_{0}, y_{0}\right)$ is any solution of fractional-order differential system with Caputo's derivative of (2.18) for $t \geq t_{0}$ such that $V\left(\tau_{0}, y_{0}-x_{0}\right)$ holds. If we define $m(t)=V(t, y(t)-\tilde{x}(t))$ for $t \geq \tau_{0}$ so that $m\left(\tau_{0}\right) \leq u_{0}$, then Theorems 5.1 and 5.2 imply that

$$
{ }^{c} D_{+}^{q} m(t) \leq G(V(t, m(t))), \quad m\left(\tau_{0}\right)=V\left(\tau_{0}, y\left(\tau_{0}\right)-\tilde{x}\left(\tau_{0}\right)\right) \leq u_{0},
$$

where the fractional-order Dini derivatives in Caputo's sense ${ }^{c} D_{+}^{q} m(t)$ and the generalized fractional-order (Dini-like) derivatives in Caputo's sense ${ }_{*}^{c} D_{+}^{q} V(t, x)$ have been used. Thus, $m(t)=V(t, y(t)-\tilde{x}(t)) \leq r\left(t, \tau_{0},\left\|y_{0}-x_{0}\right\|\right)$, where $r$ is the maximal solution of the fractional comparison system of (5.12) with Caputo's derivative. Let the null solution of the fractional comparison system of (5.12) with Caputo's derivative be stable. Given any $\epsilon>0$, since $V$ is positive definite and $a \in \mathcal{K}$ by (i) we have

$$
\begin{gathered}
a(\|y-\tilde{x}\|) \leq V(t, y-\tilde{x}) \quad \text { for }(t, \tilde{x}),(t, y) \in \mathbb{R}_{+} \times \mathbb{R}^{n}, \\
V(t, y(t)-\tilde{x}(t)) \leq r\left(t, \tau_{0},\left\|y_{0}-x_{0}\right\|\right)<a(\epsilon) \quad \text { for } t \geq \tau_{0} \text { provided that }\left\|y_{0}-x_{0}\right\|<\delta\left(\epsilon, \tau_{0}\right) .
\end{gathered}
$$

Hence

$$
V(t, y(t)-\tilde{x}(t)) \leq r\left(t, \tau_{0},\left\|y_{0}-x_{0}\right\|\right)<a(\epsilon) .
$$

Since $a^{-1}$ exists, we have $\|y(t)-\tilde{x}(t)\|<\epsilon$ and $\left\|y_{0}-x_{0}\right\|<\delta\left(\epsilon, \tau_{0}\right)$. Therefore, $y\left(t, \tau_{0}, y_{0}\right)$ the solution of fractional-order differential equation of (2.18) with Caputo's derivative is stable with respect to $\tilde{x}(t)=x\left(t-\eta, t_{0}, x_{0}\right)$, where $x\left(t, t_{0}, x_{0}\right)$ is any solution of the fractional-order 
differential equation of (2.16) with Caputo's derivative. If the null solution of the fractional comparison system of (5.12) with Caputo's derivative is asymptotically stable, then

$$
a(\|y(t)-\tilde{x}(t)\|) \leq r\left(t, \tau_{0},\left\|y_{0}-x_{0}\right\|\right) \quad \text { holds for } t \geq \tau_{0},\left\|y_{0}-x_{0}\right\| \text { small enough. }
$$

That implies

$$
\lim _{t \rightarrow \infty}\left\|y\left(t, \tau_{0}, y_{0}\right)-x\left(t-\eta, t_{0}, x_{0}\right)\right\|=0
$$

since $a \in \mathcal{K}$. Hence, $y\left(t, \tau_{0}, y_{0}\right)$ the solution of fractional differential equation (2.18) is asymptotically stable with respect to $\tilde{x}(t)=x\left(t-\eta, t_{0}, x_{0}\right)$, where $x\left(t, t_{0}, x_{0}\right)$ is any solution of the system (2.16). Since $V$ is decrescent and $b \in \mathcal{K}$ by (i) we have

$$
V(t, y-\tilde{x}) \leq b(\|(y-\tilde{x})\|) \quad \text { for }(t, \tilde{x}),(t, y) \in \mathbb{R}_{+} \times \mathbb{R}^{n}
$$

and the choice of $\delta=\delta(\epsilon)$ is independent of $\tau_{0}$.

Thus, uniform stability and uniform asymptotic stability of the fractional comparison system of (5.12) with Caputo's derivative imply the corresponding uniform stability and uniform asymptotic stability of the fractional solution of (2.18) in Caputo's sense with respect to $\tilde{x}(t)=x\left(t-\eta, t_{0}, x_{0}\right)$, where $x\left(t, t_{0}, x_{0}\right)$ is any solution of the fractional system of (2.16) in Caputo's sense. Hence, $x\left(t, t_{0}, x_{0}\right)$ is any solution of the fractional system of (2.16) in Caputo's sense that is uniformly stable and uniformly asymptotically stable.

\section{Acknowledgments}

This work has been supported by The Scientific and Technological Research Council of Turkey and Department of Mathematics Yeditepe University.

\section{References}

[1] M. Caputo, "Linear models of dissipation whose Q is almost independent, II," Geophysical Journal of the Royal Astronomical Society, vol. 13, pp. 529-539, 1967.

[2] A. A. Kilbas, H. M. Srivastava, and J. J. Trujillo, Theory and Applications of Fractional Differential Equations, North-Holland, Amsterdam, The Netherlands, 2006.

[3] F. Brauer and J. Nohel, The Qualitative Theory of Ordinary Differential Equations, W.A. Benjamin, New York, NY, USA, 1969.

[4] V. Lakshmikantham and S. Leela, Differential and Integral Inequalities, vol. 1, Academic Press, New York, NY, USA, 1969.

[5] V. Lakshmikantham, S. Leela, and A. A. Martynyuk, Stability Analysis of Nonlinear Systems, vol. 125 of Monographs and Textbooks in Pure and Applied Mathematics, Marcel Dekker, New York, NY, USA, 1989.

[6] V. Lakshmikantham, S. Leela, and J. Vasundhara Devi, Theory of Fractional Dynamic Systems, Cambridge Scientific, Cambridge, UK, 2009.

[7] M. D. Shaw and C. Yakar, "Stability criteria and slowly growing motions with initial time difference," Problems of Nonlinear Analysis in Engineering Systems, vol. 1, pp. 50-66, 2000.

[8] I. Podlubny, Fractional Differential Equations, vol. 198 of Mathematics in Science and Engineering, Academic Press, San Diego, Calif, USA, 1999.

[9] V. Lakshmikantham and A. S. Vatsala, "Differential inequalities with initial time difference and applications," Journal of Inequalities and Applications, vol. 3, no. 3, pp. 233-244, 1999. 
[10] S. G. Samko, A. A. Kilbas, and O. I. Marichev, Fractional Integrals and Derivatives: Theory and Applications, Gordon and Breach, Yverdon, Switzerland, 1993.

[11] M. D. Shaw and C. Yakar, "Generalized variation of parameters with initial time difference and a comparison result in term Lyapunov-like functions," International Journal of Non-Linear Differential Equations-Theory Methods and Applications, vol. 5, pp. 86-108, 1999.

[12] C. Yakar, "Boundedness criteria with initial time difference in terms of two measures," Dynamics of Continuous, Discrete E Impulsive Systems. Series A, vol. 14, supplement 2, pp. 270-274, 2007.

[13] C. Yakar, "Strict stability criteria of perturbed systems with respect to unperturbed systems in terms of initial time difference," in Complex Analysis and Potential Theory, pp. 239-248, World Scientific, Hackensack, NJ, USA, 2007.

[14] C. Yakar and M. D. Shaw, "A comparison result and Lyapunov stability criteria with initial time difference," Dynamics of Continuous, Discrete E Impulsive Systems. Series A, vol. 12, no. 6, pp. 731-737, 2005.

[15] C. Yakar and M. D. Shaw, "Initial time difference stability in terms of two measures and a variational comparison result," Dynamics of Continuous, Discrete $\mathcal{E}$ Impulsive Systems. Series A, vol. 15, no. 3, pp. 417-425, 2008.

[16] C. Yakar and M. D. Shaw, "Practical stability in terms of two measures with initial time difference," Nonlinear Analysis: Theory, Methods E Applications, vol. 71, no. 12, pp. e781-e785, 2009.

[17] V. Lakshmikantham and A. S. Vatsala, "Basic theory of fractional differential equations," Nonlinear Analysis: Theory, Methods \& Applications, vol. 69, no. 8, pp. 2677-2682, 2008. 


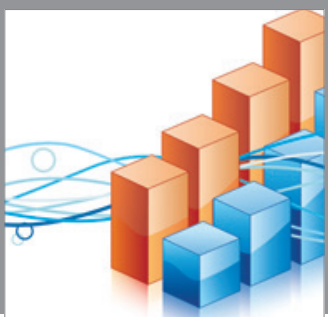

Advances in

Operations Research

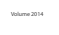

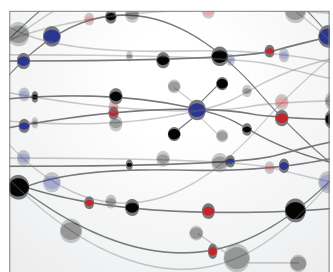

\section{The Scientific} World Journal
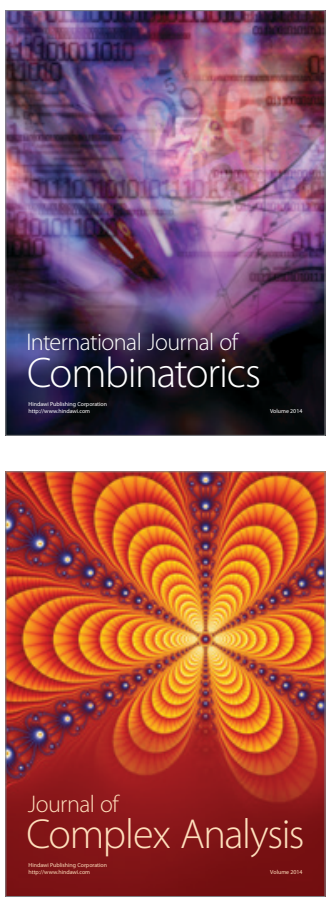

International Journal of

Mathematics and

Mathematical

Sciences
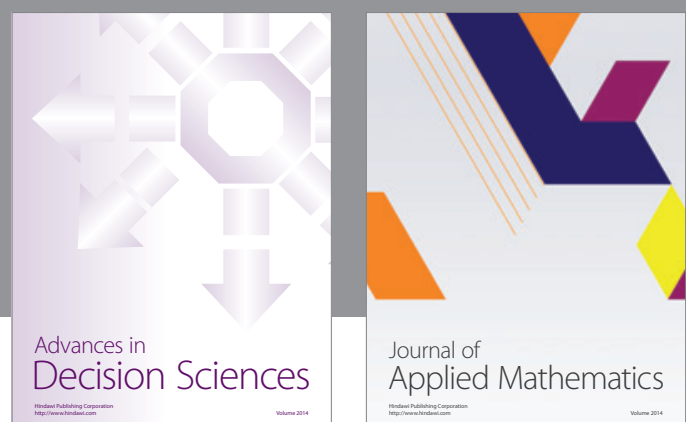

Journal of

Applied Mathematics
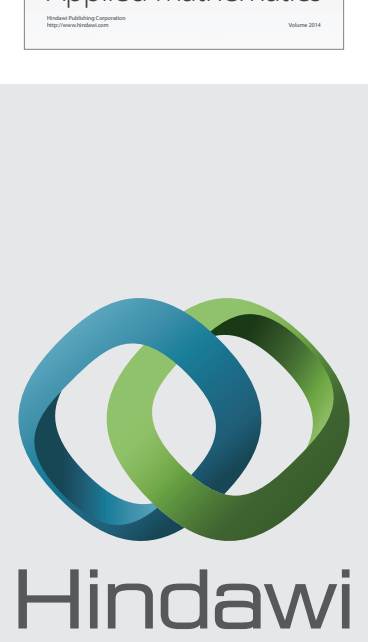

Submit your manuscripts at http://www.hindawi.com
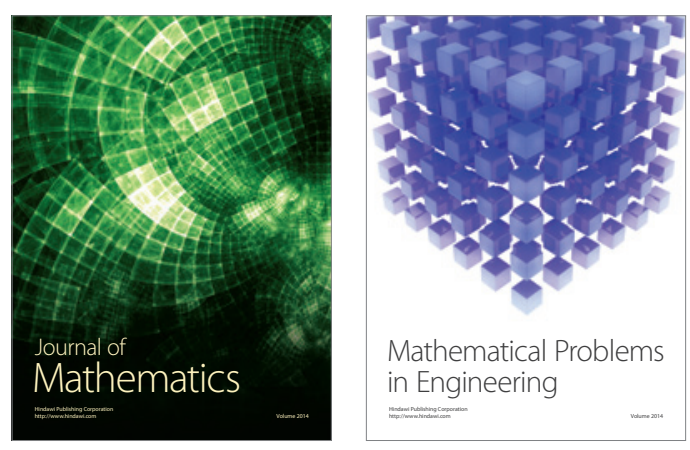

Mathematical Problems in Engineering
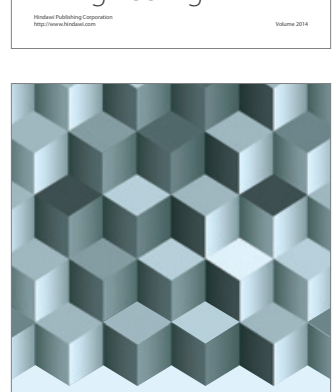

Journal of

Function Spaces
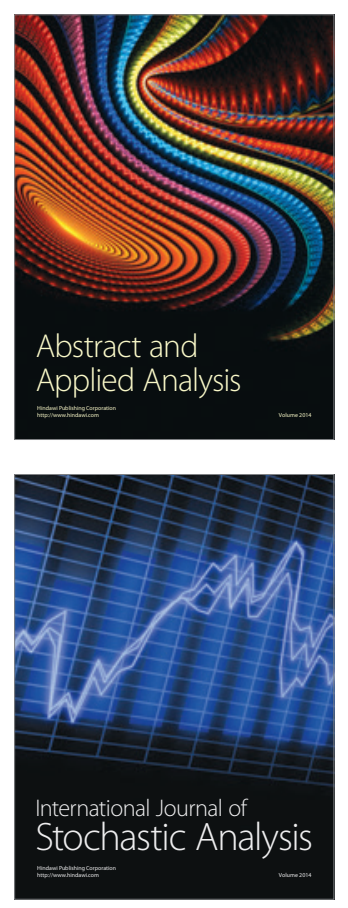

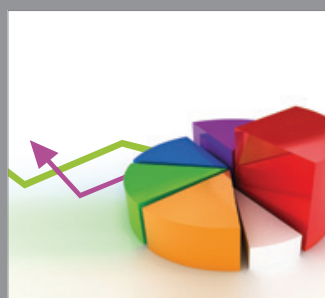

ournal of

Probability and Statistics

Promensencen
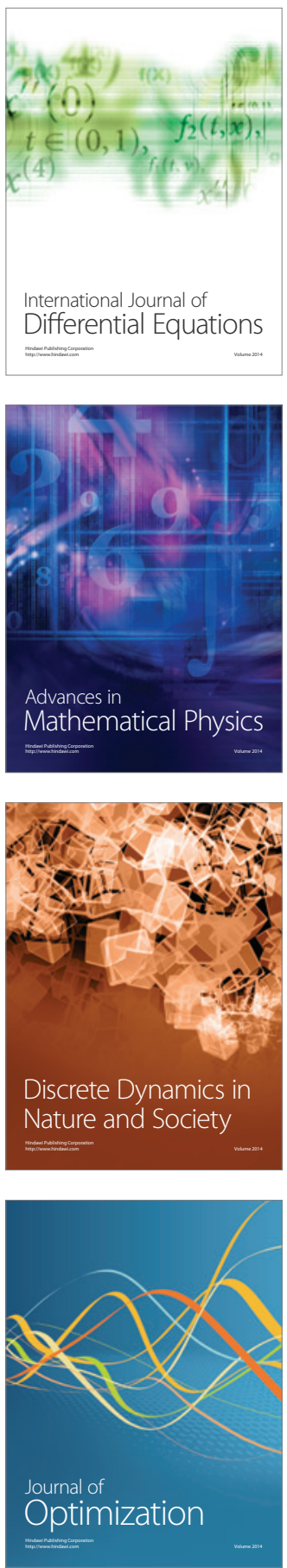